\title{
Implementation and Evaluation of Multiple Adaptive Control Technologies for a Generic Transport Aircraft Simulation
}

\author{
Stefan F. Campbell ${ }^{*}$ and John T. Kaneshige ${ }^{\dagger}$ \\ SGT and NASA, Moffett Field, CA, 94035 \\ Nhan T. Nguyen ${ }^{*}$ and Kalmanje S. Krishnakumar ${ }^{\S}$ \\ NASA Ames Research Center, Moffett Field, CA, 94035
}

\begin{abstract}
Presented here is the evaluation of multiple adaptive control technologies for a generic transport aircraft simulation. For this study, seven model reference adaptive control (MRAC) based technologies were considered. Each technology was integrated into an identical dynamic-inversion control architecture and tuned using a methodology based on metrics and specific design requirements. Simulation tests were then performed to evaluate each technology's sensitivity to time-delay, flight condition, model uncertainty, and artificially induced cross-coupling. The resulting robustness and performance characteristics were used to identify potential strengths, weaknesses, and integration challenges of the individual adaptive control technologies.
\end{abstract}

\section{Introduction}

THE Integrated Resilient Aircraft Control (IRAC) project is a part of the Aviation Safety Program under the Aeronautics Research Mission Directorate (ARMD) at NASA. A key focus of this project is to research the use of adaptive control technologies as a risk-mitigating tool for off-nominal aircraft flight. In a traditional gainscheduled design approach, the flight controller is designed by treating the aircraft's flight envelope as a discrete space. Controls engineers then use traditional linear control techniques to shape the handling qualities of the aircraft at each of these discrete locations. In an off-nominal scenario, this design approach may breakdown as a result of the introduction of large model uncertainties. Even in the case of a relatively robust gain-scheduled controller, aircraft performance may be severely impacted. The goal of an adaptive flight controller is to then augment the baseline controller such that unexpected model uncertainties may be compensated for rapidly. If general aircraft safety is not the focus, the improvements associated with the inclusion of adaptive control technologies can be performance based; an adaptive controller may help compensate for the modeling errors inherent in the control designer's analytical tools.

Irrespective of the motivation, a pilot will be interacting with the adaptively augmented flight control system. Given this fact, this paper provides an overview of a preliminary study to investigate both the analytical and qualitative characteristics of an adaptive flight controller. This study was performed as a precursor to pilot-in-theloop evaluations in which handling qualities would be assessed in a high-fidelity simulation environment. For this work, a subset of adaptive control technologies was selected for study. These technologies were then tuned using a fixed time-delay metric for a nominal aircraft. Finally, the resulting tuned controllers were evaluated under a variety of failure and damage scenarios in order to assess the robustness features of each technology.

\section{Adaptive Control Technologies}

This work does not aim to cover in detail the development and/or theoretical justification for any one adaptive control technology, nor does this work intend to survey the full breadth of the adaptive control literature. Rather, this

\footnotetext{
* Controls Engineer, Mail Stop 269-1, AIAA Member.

${ }^{\dagger}$ Computer Engineer, Intelligent Systems Division, Mail Stop 269-1, AIAA Member.

* Research Scientist, Intelligent Systems Division, Mail Stop 269-1, AIAA Associate Fellow.

$\S$ Principal Investigator, Intelligent Systems Division, Mail Stop 269-1, AIAA Associate Fellow.
} 
section will first discuss model reference adaptive control (MRAC) in very broad terms. This approach to adaptive flight control has many variations, but has seemingly come to dominate the focus of the adaptive controls community of recent. Second, this section will present the baseline controller for use in this study and provide relevant discussion for testing multiple technologies in a coherent framework. Finally, a high-level discussion of the adaptive control technologies to be explored in this study will be given.

\section{A. General MRAC}

The general concept for adaptive control can be illustrated with a simple linear system model, as shown below:

$$
\dot{x}=A x+B u .
$$

This represents the linear model that the controls engineer would nominally use to design a feedback control law $u$. That feedback control law, depending on the type of control system chosen, may then be generically written as

$$
u=K_{x}^{T} x+K_{r}^{T} r
$$

where $r$ is the pilot provided reference signal. The closed loop system has then been designed to behave dynamically as

$$
\dot{x}=\left(A+K_{x}^{T}\right) x+B K_{r}^{T} r .
$$

This defines the adaptive control reference model and is generally written as the following:

$$
\dot{x}_{m}=A_{m} x_{m}+B_{m} r .
$$

The actual aircraft system in (1), however, includes uncertainty. The true aircraft dynamics are modeled as the following:

$$
\dot{x}=A_{T} x+B \Lambda(u+f) .
$$

Here we have introduced the true " $A$ " matrix, $A_{T}$, and an unknown matrix $A$ that scales the $B$ matrix in (1). Additionally, a set of non-linear uncertainties is introduced with $f$, which is modeled as the following:

$$
f=W^{T} \phi(x)+\varepsilon(x)
$$

Here, the nonlinear uncertainty is parameterized by a set of basis functions $\phi(x)$ and an ideal set of weights, $W$, and an associated approximation error $\varepsilon(x)$. The motivation for adaptive control is to compensate for the uncertainties in the system model such that the system behaves as in (4). To this end, it is assumed that there exist ideal gains (denoted with an ${ }^{*}$ ) such that the following statements can be made true:

$$
\begin{aligned}
& \exists K_{x}^{*}, A_{T}+B \Lambda K_{x}^{*} T=A_{m} \\
& \exists K_{r}^{*}, B \Lambda K_{r}^{* T}=B_{m}
\end{aligned}
$$

To evaluate the difference between the true system and the reference model, we subtract (5) from (4) and substitute in (2) to obtain the following error dynamics:

$$
\dot{e}=\dot{x}_{m}-\dot{x}=A_{m}\left(x_{m}-x\right)+B \Lambda\left[\left(K_{x}^{* T}-K_{x}^{T}\right) x+\left(K_{r}^{* T}-K_{r}^{T}\right) r-f\right]=A_{m} e+B \Lambda\left[D_{x}^{T}+D_{r}^{T} r-f\right]
$$

In (7), $D$ terms have been introduced to account for the error between the ideal weights and the chosen control law weights. The adaptive control formulation is then designed to adjust the control law in (2) such that the uncertainties (the $D$ and $f$ terms in (8)) are canceled. The adaptive control law may then be formulated as: 


$$
u=K_{x}^{T} x+K_{r}^{T} r-\hat{D}_{x}^{T} x-\hat{D}_{r}^{T}+\hat{W}^{T} \phi(x)
$$

The real-time parameter estimates are then determined from an adaptive control law. In the context of MRAC, these adaptive control laws are defined using Lyapunov stability proofs and the system's error dynamics, which are presented in (10).

$$
\dot{e}=A_{m} e+B \Lambda\left[\Delta D_{x}^{T}+\Delta D_{r}^{T} r-\Delta W^{T} \phi(x)-\varepsilon(x)\right]
$$

In (10), $\Delta$ denotes the difference between the true and the estimated parameter values, as in $\Delta D_{x}=D_{x}-\hat{D}_{x}$. The general MRAC adaptive control laws are the following:

$$
\begin{aligned}
& \hat{D}_{x}=-\Gamma_{x} x e^{T} P B \\
& \hat{D}_{r}=-\Gamma_{r} r e^{T} P B \\
& \hat{W}=\Gamma_{\phi} \phi(x) e^{T} P B
\end{aligned}
$$

The $\Gamma$ terms are the designer selected adaptive gains and the matrix $P$ is a symmetric, positive definite matrix defined using the well-known Lyapunov equation

$$
P A_{m}+A_{m}^{T} P=-Q
$$

where $Q$ is also a designer chosen symmetric, positive definite matrix. The adaptive laws in (11) can, however, be simplified to a single complete statement by redefining the control law in (9) as the following:

$$
u=K_{x}^{T} x+K_{r}^{T} r-u_{a d}
$$

The adaptive control component can then be written succinctly as

$$
u_{a d}=\hat{\bar{W}}^{T} \beta_{a d}\left(x_{c}\right) .
$$

Here we have combined the estimated parameters of (9) into a single matrix of unknown weights multiplied by a single vector of basis functions $\beta_{a d}$. Additionally, the vector $x_{c}$ is a concatenation of vectors $x$ and $r$. Given this notation, the adaptive control law of (11) is then simplified to

$$
\dot{\bar{W}}=-\Gamma \beta_{a d}\left(x_{c}\right) e^{T} P B
$$

Regardless of the notation chosen, the adaptive system error dynamics are uniformly ultimately bounded (UUB) outside of the domain

$$
e:\|e\| \leq \frac{2\|P B\| \lambda_{\max }(\Lambda) \varepsilon_{b}}{\lambda_{\min }(Q)} .
$$

In (16), $\lambda$ denotes an eigenvalue and $\varepsilon_{b}$ is the bound of the approximation error norm $(\varepsilon(x))$.

This result (though not fully derived or justified here) shows that the error between the reference model and the true system will move within a neighborhood of 0 and remain there without necessarily converging to 0 . The size of this neighborhood is dictated by the designer chosen variable $Q$, the estimation uncertainty bound $\varepsilon_{b}$, and the form of the error dynamics ( $A_{m}$ impacts the norm of $P$ through the Lyapunov equation). In general, it is desired to keep the neighborhood about 0 as small as possible. Finally, the result in (16) does not provide information on the performance of the adaptive parameters themselves. As such, these parameters must be externally bounded to prevent parameter drift and the well-known bursting phenomenon; this is usually accomplished via inclusion of the projection operator in (11) or (14). 


\section{B. Baseline Controller - Dynamic Inversion}

The value of the previous section is in isolating several key variables for evaluating adaptive control technologies, including the baseline control law chosen in (2) and the basis functions $\phi(x)$. MRAC based adaptive control technologies generally consist of variations and modifications to the adaptive control laws that provide the parameter estimates. However, overall system performance is a function of both the adaptive control laws and the aforementioned components. In particular, the baseline controller and system model dictate the size of the unknown parameters $D$ in (6) and the basis functions $\phi(x)$ determine the approximation error $\varepsilon(x)$. Both of these parameters in turn determine the size of the $U U B$ domain. For developing a consistent study, it is then necessary that each adaptive controller augment the same baseline controller (including gains $K_{x}$ and $K_{r}$ ) using an identical set of basis functions. Moreover, by using an identical baseline controller, the $B$ matrix in (11) and the reference model in (4) are consistent amongst the adaptive control technologies. With this established, the flight test research presented in [7] and [8] is used as the motivation for selecting a baseline controller and an appropriate set of basis functions.

\section{Baseline Controller}

The baseline controller for this work is a full non-linear dynamic inversion controller based in large part on [7], [9], and [10]. This control architecture is chosen for several key reasons. First, this control approach can be effectively implemented as evinced by the recent selection of a dynamic inversion controller for the F-35 aircraft. Second, a dynamic inversion controller can often be more cost and time effective to develop than a traditional gain scheduled controller, especially in the context of a research study. This ultimately facilitates a much more rapid testing and evaluation of new control technologies across a broad range of flight conditions.

The general (and well known) rigid body dynamics for an aircraft (assumed undamaged) are presented below:

$$
\tau=I \dot{\omega}+\omega \times I \omega .
$$

Here, $\omega$ represents a vector of the roll $(p)$, pitch $(q)$, and yaw $(r)$ rates. By modeling the torques on the aircraft using traditional aerodynamic stability derivatives (which includes $p, q$, and $r$ aerodynamic derivatives), (17) may be decomposed into the following convenient form:

$$
\dot{\omega}=A \omega+G z+B u .
$$

For this decomposition, the matrices $A, G$, and $B$ are time-varying, but for simplicity this time dependency is not expressed. Moreover, the aircraft's control allocation tables are incorporated into (18) such that the control vector $u$ is $\in \mathfrak{R}^{3 \mathrm{X} 1}$ and represents the three, non-dimensional lateral, longitudinal, and directional control signals (limited from -1 to 1). The vector $z$ represents a non-linear combination of $\omega$, specifically $p q, q r, r p,\left(p^{2}-q^{2}\right),\left(r^{2}-p^{2}\right)$, and $\left(q^{2}\right.$ $-r^{2}$ ), as well as a bias term (which accounts for the $\tau$ dependence on slowly changing variables such as angle of attack and side-slip).

A general non-linear dynamic inversion control law is then

$$
u=\hat{B}^{-1}(\dot{\omega} d-\hat{A} \omega-\hat{G} z) .
$$

For clarity, over-hats have been used to denote that parameters in the dynamic inversion control law are estimated values; these are not adaptively estimated parameters, but rather parameters extracted from online look-up tables based upon the aircraft's current flight condition. Additionally, the subscript $d$ denotes a desired value.

Using (19), the baseline, 3-axis control architecture is designed using pilot stick inputs as roll and pitch rate commands; the pilot pedal inputs are taken as commands to sideslip. For the roll and pitch channels, simple PI controllers are used to generate roll and pitch desired acceleration commands.

For the directional axis, a two stage dynamic inversion is employed, similar to the work done in [11]. Specifically, in [11] the dynamic inversion of (19) inverts the fast dynamics and an additional slow dynamic inversion is employed to generate the necessary roll, pitch, and yaw rate commands. For this work, a slow inversion is employed only on the directional axis with a proportional outer loop controller. This generates an equivalent yawrate command for the inner loop fast dynamic inversion, which includes a PI controller (as in the roll and pitch channels).

For the slow dynamic inversion, the following well-known relationship is used as a starting point for derivation: 


$$
\begin{aligned}
\dot{\beta}=p \sin (\alpha)-r \cos (\alpha)+ & \left(1 / m V_{t}\right)\left[D \sin (\beta)+Y \cos (\beta)-X_{T} \cos (\alpha) \sin (\beta)+m g(\cos (\alpha) \sin (\beta) \sin (\theta)\right. \\
+ & \cos (\beta) \sin (\phi) \cos (\theta)-\sin (\alpha) \sin (\beta) \cos (\phi) \cos (\theta))]
\end{aligned}
$$

The expression in (20) is simplified to a more tractable expression:

$$
\dot{\beta} \approx p \sin (\alpha)-r \cos (\alpha)+\left(g / V_{t}\right) \cos (\theta) \sin (\phi) .
$$

The yaw rate command is then determined from (21), as shown in (22).

$$
r_{\text {com }}=-\left(\dot{\beta}_{d}-p \sin (\alpha)-\left(g / V_{t}\right) \cos (\theta) \sin (\phi)\right) / \cos (\alpha)
$$

To further augment this system, a command filter is used to generate a feed-forward term for each axis of the fast dynamic inversion inner loop, as well as the slow outer loop sideslip controller. This command filter serves as a component of the adaptive reference model. The complete architecture is presented below in Fig. 1 and Fig. 2.

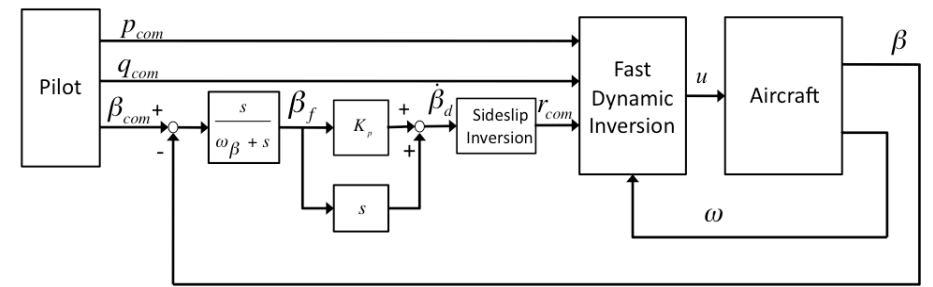

Fig. 1 High-level base-line control architecture. Here the dependency of the sideslip inversion on additional states $\left(\alpha, \theta, \phi, p\right.$, and $\left.V_{t}\right)$ is not illustrated.

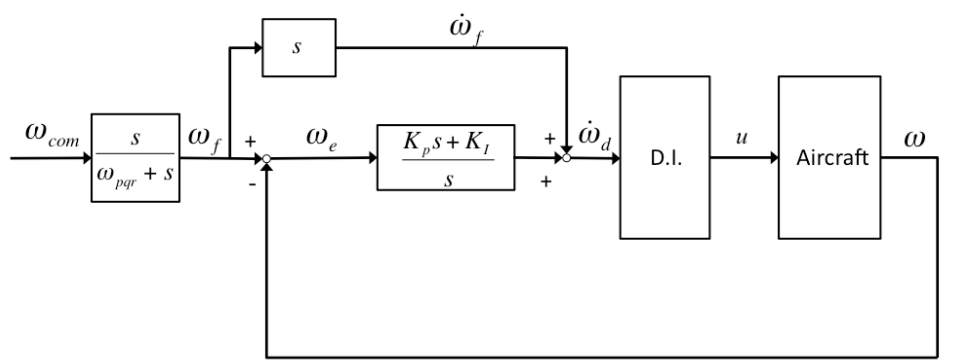

Fig. 2 The inner-loop fast dynamics controller. The D.I. block contains the equation presented in (19).

The desired angular accelerations for this architecture (as diagramed in Fig. 1) are given below in (23).

$$
\dot{\omega}_{d}=K_{p}\left(\omega_{f}-\omega\right)+K_{I} \int_{0}^{t}\left(\omega_{f}-\omega\right) d \tau+\dot{\omega}_{f}
$$

Here, the output of the command filter is expressly represented with a subscript $f\left(\omega_{f}^{T}=\left[\begin{array}{lll}p_{f} & q_{f} & r_{f}\end{array}\right]\right)$. Moreover, it should be noted that both $K_{p}$ and $K_{I}$ are diagonal, $3 \times 3$ matrices. As a final note on the general architecture, the pilots commanded pitch-rate is augmented by the level turn-compensation term $g \sin ^{2} \phi / V \cos \phi$. Qualitatively, this helps keep the nose of the aircraft up during bank and roll maneuvers.

With a dynamic inversion controller it is necessary to generate a reasonable aircraft model to estimate the inversion parameters. In a simulated study, a dynamic inversion controller can be unrealistically robust by circularly using the simulated aircraft model to provide the inversion parameters. In this study we use an aircraft model based on actual wind-tunnel data for the simulation. The dynamic inversion controller model is, however, generated using a numerical vortex-lattice code package (see section IV). This ensures that the control system operates with a realistically uncertain model. 


\section{Basis Functions}

As aforementioned, an identical set of regressor functions ( $\beta\left(x_{c}\right)$ in the section II.A) was chosen for each adaptive technology. The basis functions used for this study are presented below.

\begin{tabular}{|c|c|}
\hline Control Channel & Regressor Vector \\
\hline Lateral & bias, $\dot{p}_{f}, \dot{q}_{f}, \dot{r}_{f} p_{f}, q_{f} r_{f}, p, r, p q, q r, \beta$ \\
\hline Longitudinal & bias, $\dot{p}_{f}, \dot{q}_{f}, \dot{r}_{f} p_{f}, q_{f} r_{f}, q, r, r p, p^{2}-r^{2}, \alpha$ \\
\hline Directional & bias, $\dot{p}_{f}, \dot{q}_{f}, \dot{r}_{f} p_{f}, q_{f}, r_{f}, p, r, p q, q r, \beta$ \\
\hline
\end{tabular}

Table 1. Adaptive Control Basis Functions by Control Channel

For this study, each control channel of the adaptive control system was treated separately. This is analogous to having a single large regressor vector and constraining certain elements of the adaptive weight matrix to be zero, which can be achieved via the well-known projection operator. For the sake of clarity it is worth connecting this section with the notations used in the previous sections. Specifically, many of the non-linear terms in the regressor vectors of Table 1 are contained in the vector $z$ of Equations (18) and (19). The regressor vectors as a whole are the basis functions $\phi(x)$ of Equation (6). From this point forward the terminology regressor vector is used to emphasize that the signals are not necessarily the typically assumed sigmoidal or radial basis functions often used in neuralnetwork based adaptive control. Analyzing Equation (6), it should be understood that the selection of $\phi(x)$ will implicitly dictate the approximation error $\varepsilon(x)$. As an example, if a null regressor vector were selected, the uncertainty term $f$ would be defined entirely by the approximation error; a null regressor vector would produce a poor approximation of $f$.

\section{Adaptive Control Technologies}

The adaptive control technologies used for this study represent a cross-section of many of the adaptive control technologies found in the academic community. To present each technology, a brief general discussion of each technology's motivation is provided. This discussion is accompanied by an abbreviated overview of how each technology modifies the general MRAC structure discussed in section II.A. In general, each technology fits into the architecture of Fig. 2 by providing an augmentation signal to the pseudo-command defined in (23). This is illustrated graphically in Fig. 3. A thorough discussion of each of these technologies can be found in the provided references.

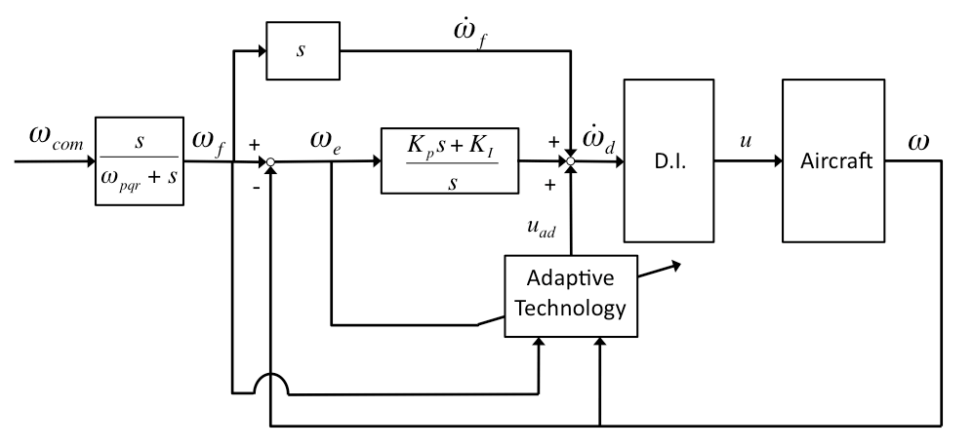

Fig. 3 General MRAC Technology Augmentation to Baseline Controller

\section{Adaptive Loop Recovery MRAC (ALR)}

The adaptive loop recovery approach to MRAC modifies the adaptive laws in (15) by adding an additional modification term. This modification term is designed to recover the loop properties (i.e. the stability margins) of the reference model. In implementation, a traditional MRAC controller modifies the system in a non-linear fashion such that stability properties cannot be accurately assessed. If the adaptive laws are linearized, however, the system will retain the reference model properties if certain conditions are satisfied. The adaptive loop recovery modification term aims towards achieving such conditions. To this end the adaptive control law is defined as the following: 


$$
\dot{\hat{\bar{W}}}=-\Gamma\left[\beta_{a d}\left(x_{c}\right) e^{T} P B-v \frac{\partial \beta\left(x_{c}\right)}{\partial x_{c}}\left(\frac{\partial \beta\left(x_{c}\right)}{\partial x_{c}}\right)^{T} \hat{\bar{W}}\right]
$$

In (24), $v$ is an additional gain term. Further detail on this control technology is provided in [12].

\section{Bounded Linear Stability MRAC (BLS)}

The Bounded Linear Stability modification to MRAC adjusts the adaptive gain in (15) to preserve the system's overall stability margins. This is achieved by evaluating a piece-wise linear approximation to the non-linear system (including adaptive control laws) and applying the LTI concept of stability margins over discrete time-windows. This modification is fully described in [13] and [14].

\section{Hybrid Adaptive MRAC (Hybrid)}

This adaptive control component combines the direct-adaptive control method of MRAC with an indirect adaptive control method. Specifically, traditional MRAC is augmented by using the recursive least squares algorithm (RLS) to update the dynamic inversion parameters in (19). This architecture is illustrated graphically in Fig. 4 and fully discussed in [10].

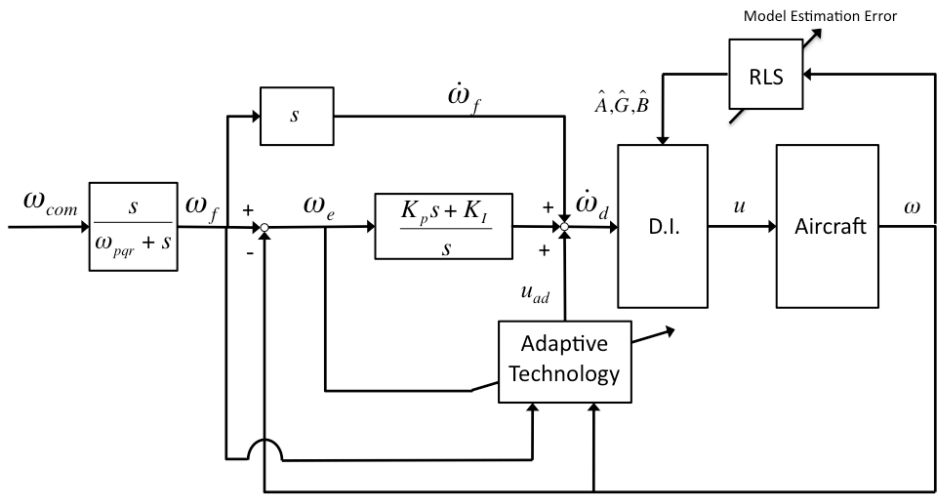

Fig. 4 Hybrid Adaptive Control Implementation

\section{4. $L_{1}$ Adaptive Control $\left(L_{1}\right)$}

The $L_{l}$ adaptive control approach has recently garnered significant attention from the controls community. In this architecture, the uncertainty of the system is approximated using large gains in the adaptive control laws. The control signal $u$ (13) is then augmented by a filtered version of the high-gain adaptive signal (which will, by virtue of the high-gain, contain high-frequency content). The error dynamics, however, are not determined from the traditional reference model (4), but instead by a predictor system. This predictor system is defined very precisely as a combination of the reference model and the difference between the filtered and unfiltered adaptive signal. Conceptually, the filter serves as a model of the amount of uncertainty the system can effectively cancel. The predictor system then provides an achievable reference signal by fully considering uncertainty that cannot be effectively canceled by limited control effectors. The resulting adaptive control signal is given as

$$
u_{a d}(s)=C(s) \bar{r}(s),
$$

where

$$
\bar{r}(t)=\hat{\bar{W}}^{T} \beta\left(x_{c}\right) .
$$

In (25), $C(s)$ represents a designer selected filter of order $n$. The $L_{l}$ predictor system is then given as the following:

$$
\dot{\hat{x}}=A_{m} \hat{x}+B_{m} r+B\left(\hat{\bar{W}}^{T} \beta\left(x_{c}\right)-u_{a d}\right)
$$


The adaptive control laws then remain as in (15) but the error term is redefined as $e=\hat{x}-x$. Further detail on this technology is provided in [15], [16], and [17]. A detailed discussion of how this architecture is implemented with respect to the previously discussed dynamic inversion is provided in [22].

\section{Optimal Control Modified MRAC (OCM)}

The optimal control modified MRAC derives new adaptive control laws using optimal control theory. The resulting adaptive control laws are as in (15) but with an additional modification term. This term ultimately balances system performance (precise tracking) with system robustness and stability. As a result of this additional modification term, larger adaptive gains are allowable. The adaptive control law for this technology is expressed below:

$$
\dot{\hat{\bar{W}}}=-\Gamma\left[\beta_{a d}\left(x_{c}\right) e^{T} P B-v \beta\left(x_{c}\right) \beta_{a d}^{T}\left(x_{c}\right) \hat{\bar{W}} B^{T} P A_{m}^{-1} B\right]
$$

The additional gain term $v$ is by definition limited [0 1 l] with 0 corresponding to optimal tracking and 1 corresponding to optimal robustness. A full description of this approach is provided in [18].

\section{Predictor-Based MRAC (PMRAC)}

Predictor-based MRAC combines a traditional MRAC architecture with a predictor system approach. Specifically, the predictor system resembles an observer where the reference model is adjoined with the error between the prediction and the true states multiplied by a gain $A_{p}$. The adaptive control laws are then formulated using a composite of two error signals: the error between the system and the reference model and the error between the system and the predictor. The predictor system is defined as:

$$
\dot{\hat{x}}=A_{p}(\hat{x}-x)+A_{m} x+B_{m} r
$$

The composite adaptive control law is then defined as

$$
\dot{\hat{W}}=-\Gamma \beta_{a d}\left(x_{c}\right) \bar{e}^{T}
$$

where

$$
\bar{e}=B^{T}\left(P e+P_{p}(\hat{x}-x)\right) .
$$

The insertion of the term $P_{p}$ requires the reapplication of the Lyapunov equation using $A_{p}$. A complete justification of this approach is presented in [19]. A detailed discussion of how this architecture is implemented with respect to the previously discussed dynamic inversion is provided in [23].

\section{7. e-Mod MRAC (IFC)}

The final adaptive control technique to be considered is a traditional MRAC control architecture operating with a small e-mod dampening term. The adaptive laws with this dampening term are presented below where $\sigma$ is an additional gain term.

$$
\begin{aligned}
& \hat{D}_{x}=-\Gamma_{x}\left(x e^{T} P B+\sigma_{x}\left\|e^{T} P B\right\| \hat{D}_{x}\right) \\
& \hat{D}_{x}=-\Gamma_{r}\left(r e^{T} P B+\sigma_{r}\left\|e^{T} P B\right\| \hat{D}_{r}\right) \\
& \hat{W}=-\Gamma_{\phi}\left(\phi(x) e^{T} P B+\sigma_{\phi}\left\|e^{T} P B\right\| \hat{W}\right)
\end{aligned}
$$

In general, this section has presented a brief overview of each of the adaptive controllers considered for this study. The interested reader should refer to the provided references for a thorough discussion of the theoretical and technical specifications of each controller. 


\section{Tuning and Metric Evaluations}

This section presents an approach for performing a preliminary tuning of each of the control architectures discussed in section II.C. In as far as the authors are aware, there is currently no generally accepted approach for tuning the design parameters (of which there can be many) for an adaptive controller. Moreover, the designers/inventors of these adaptive control technologies do not offer precise tuning methodologies for all of the free parameters, in the best cases, rules of thumb are offered for some of the free parameters. Some work has been done on this topic, as in [20], however, a clear technique for selecting these design parameters to work across all flight conditions (not just at a single flight condition using a linearized model) is not readily known to the authors. Moreover, many of the design methodologies proposed are complicated and require using numerical techniques to solve optimization problems or the determination of mathematically non-trivial metrics using approximate system models. Given this, a more practical approach was taken here to facilitate the evaluation and investigation of multiple adaptive control technologies in a time-efficient manner. Presented first are the metrics used for evaluation followed by the tuning procedure itself.

\section{A. Tuning Metrics}

The metrics considered here are generally taken from the discussion presented in [21]. In this work, several performance metrics were discussed, including transient performance metrics, steady-state performance metrics, and cross-coupling metrics.

For the evaluation of transient and steady-state performance, the following normalized metrics were proposed, respectively (note that the metric subscript numbering is kept consistent with the original publication):

$$
M_{4}=\frac{\left\|x(t)-x_{m}(t)\right\|_{L_{x}}}{\left\|x_{m}(t)\right\|_{L_{x}}}
$$

and

$$
M_{5}=\frac{\left\|x(t)-x_{m}(t)\right\|_{L_{2}}}{\left\|x_{m}(t)\right\|_{L_{2}}} .
$$

For these metrics, the following norm definitions are employed:

$$
\|x(t)\|_{L_{\infty}} \equiv \max _{t \in\left[t_{o}, t_{f}\right]}\|x(t)\|
$$

and

$$
\|x(t)\|_{L_{2}} \equiv \sqrt{\int_{t_{o}}^{t_{f}}\|x(t)\|^{2} d t} .
$$

To evaluate cross-coupling, the following three metrics were considered:

$$
\begin{aligned}
& M_{8}=\sqrt{\int_{t_{o}}^{t_{t}} \delta_{e}^{2}(t)\left(p^{2}(t)+r^{2}(t)\right) d t}, \\
& M_{9}=\sqrt{\int_{t_{o}}^{t_{t}} q^{2}(t)\left(\delta_{a}^{2}(t)+\delta_{r}^{2}(t)\right) d t},
\end{aligned}
$$

and

$$
M_{10}=\sqrt{\int_{t_{o}}^{t_{t}}\left[p_{e}(t) q_{e}(t)+p_{e}(t) r_{e}(t)+q_{e}(t) r_{e}(t)\right]^{2} d t} .
$$


A full description of the motivation for these metrics, as aforementioned, is provided in [21]. In practice, the $M_{5}$ was the proved to be the easiest to implement and evaluation. As such, the results presented in subsequent sections use this metric almost exclusively. Additionally, it should be noted that discrete forms of the norms presented in (34) and (35) are employed in practice.

\section{B. Tuning Methodology}

To tune each adaptive controller, a general design methodology was established based on a set of design requirements. First, each adaptive technology would have its free-parameters held constant for all flight conditions in the proposed experiment (i.e. the design parameters are not themselves gain scheduled). Second, each adaptive control technology had to be robust to a .05 second time-delay. This design requirement is based on the authors experience with flight-test hardware for advanced control concepts. The time-delay is illustrated graphically for the baseline dynamic inversion controller in Fig. 5.

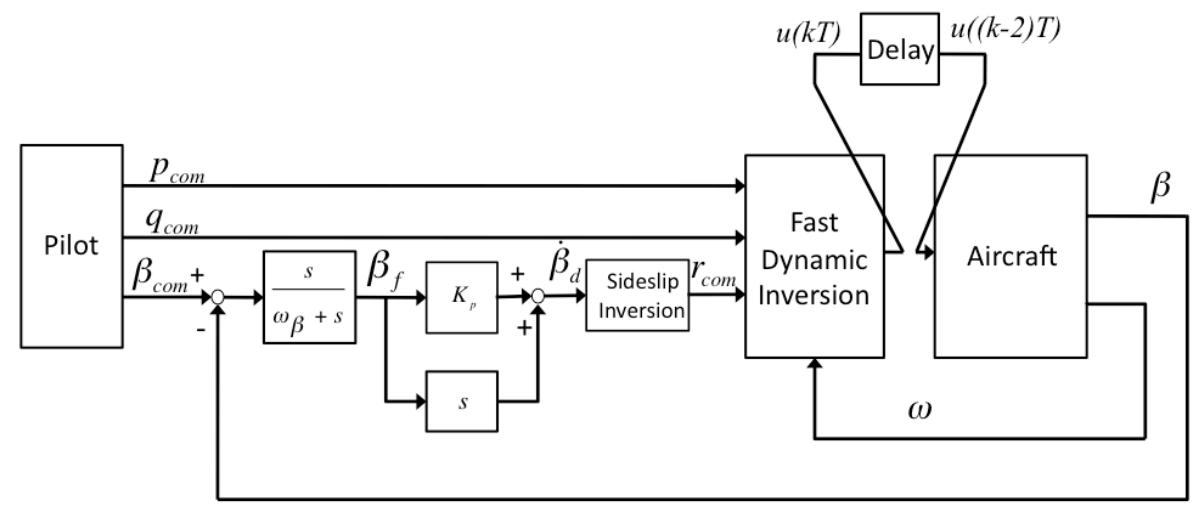

Fig. 5 Dynamic Inversion Controller with Time-Delay and a control period of $T$.

To tune each controller, the controller was evaluated using a pilot step input to each control axis. The simulated pilot input is illustrated in Fig. 6 below. Two flight conditions were considered: an altitude of 10,000 ft. and an IAS of $250 \mathrm{Kts}$. and an altitude of 10,000 ft. and an IAS of $140 \mathrm{Kts}$. In the latter scenario, the aircraft was in a fulllanding configuration with full flaps and landing gear deployed. Regardless of the flight condition, the controller was simulated with the aforementioned time-delay of .05 seconds.
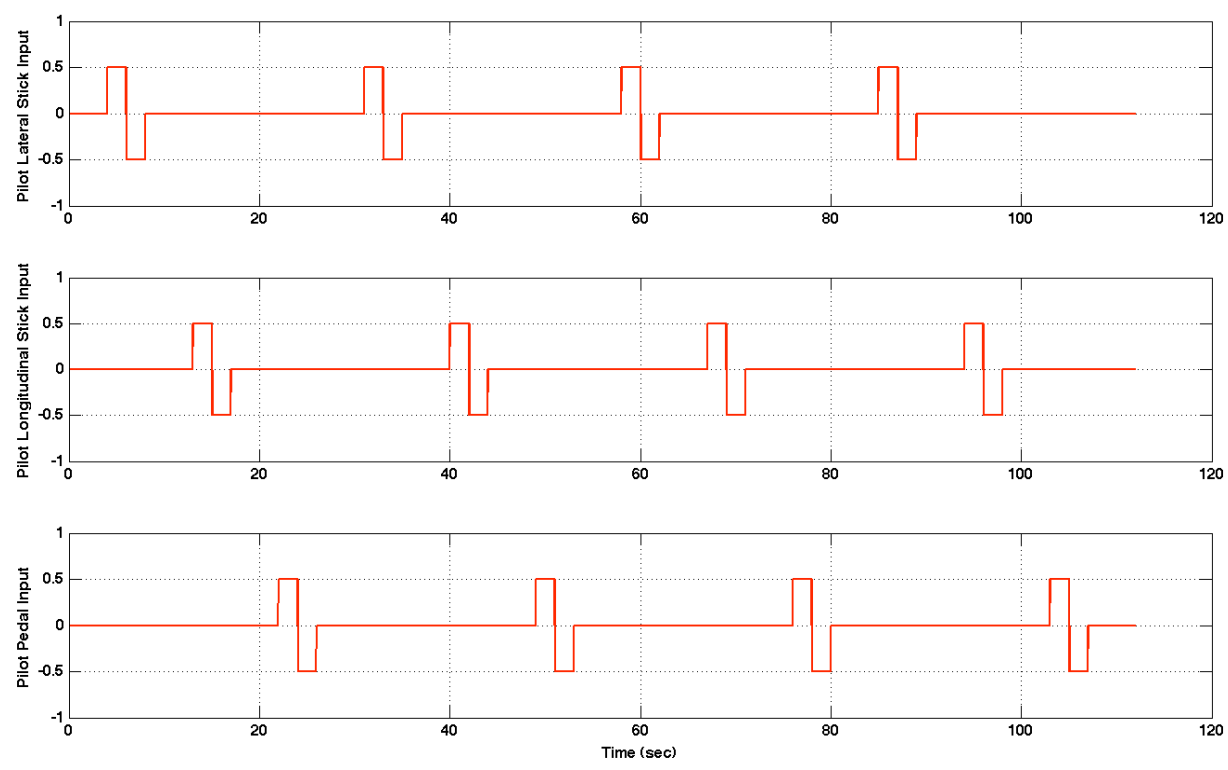

Fig. 6 Pilot Doublet Input for non-dimensional stick and rudder pedals (control input from -1 to 1). 
The design methodology was to grossly tune the free-design parameters to achieve improved performance with respect to the baseline controller. The measure of improved performance was here confined to metric $M_{5}$ (33); the remaining metrics were used primarily for sensitivity analysis. To find each controller tuning, the aforementioned scenarios were simulated and then the resulting data was analyzed to measure the performance. The adaptive gain was, in most cases, taken as the primary tuning parameter and increased as much as possible to achieve a reduced value for $M_{5}$. In this approach, the time-delay requirement provides a robustness constraint that prohibits excessively large gains, which can be unrealistically successful in idealized simulation environments.

\section{Simulation Description}

This section presents a brief overview of the aircraft models used for this study. Greater detail on these models can be found in the provided references.

\section{A. General Transport Model}

The aircraft model used for this study is the twin-engine, NASA Langley Research Center developed Generic Transport Model (GTM) [4], [5]. A 5.5\% sub-scale model of the GTM was built and used to collect aerodynamic data in a wind tunnel. This data was modified at the Ames Research Center to account for Reynolds Number affects in order to adequately model a full-scale version of the GTM. The general GTM is presented below in Fig. 7.

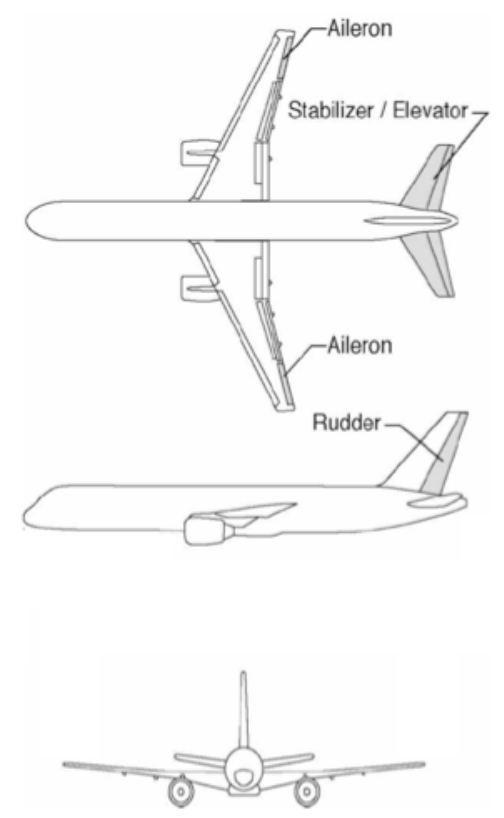

Fig. 7 Generic Transport Model

\section{B. Vortex Lattice Code Generic Transport Model}

In addition to a wind-tunnel based model of the GTM, damage models of the aircraft were approximated using a modified vortex-lattice code base developed at the Ames Research Center [6]. Detailed geometric models of the aircraft were generated for various damage configurations, including losses to portions of the wing, horizontal tail, and vertical tail. These detailed geometric models were then used with the aforementioned numerical package to generate estimates of the aircraft's aerodynamic coefficients and stability derivatives. These detailed geometries also provided inertia data for the full-scale undamaged aircraft and the various damage models. 


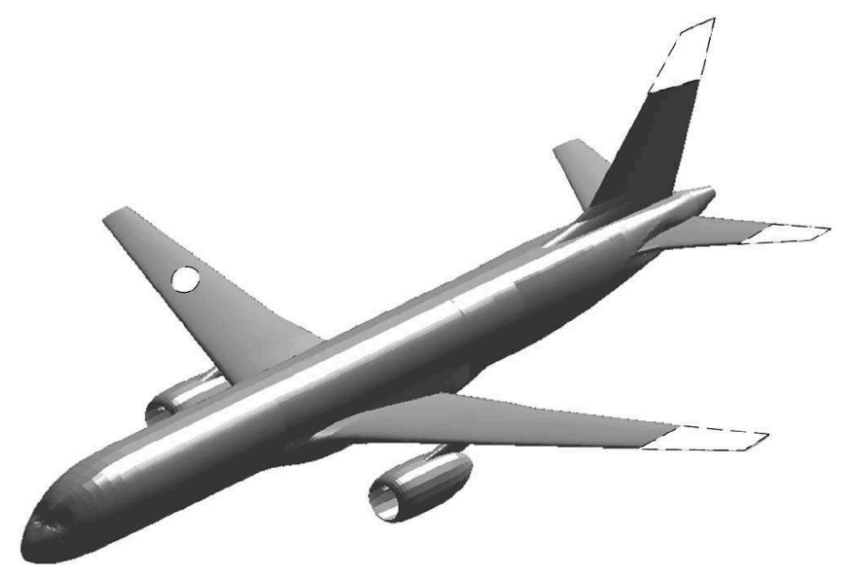

Fig. 8 Detailed Geometry of GTM Under Various Damage Conditions

\section{Simulation Environment}

The simulator used for this study features full non-linear, rigid-body equations of motion but does not model auxiliary systems, such as fuel systems and physical input/output force-feedback controls (such as throttles and sidesticks). This $G N U \mathrm{C}$ based flight simulator offers several key features: pilot inputs can be scripted and run repeatedly without variation from a desktop computer, the simulation and control frequency can be adjusted as necessary, and the overall simulation can be scripted with different setup files specifying simulation parameters. Additionally, using a $C$ based flight simulator requires that finite precision and fixed integration time steps be employed; this more accurately approximates the real-time systems that any adaptive control system would actually run in a real-world application.

\section{Simulated Damage and Failure Conditions}

In this study four failures types are considered: the artificial inclusion of a scaled $B$ matrix and a null A matrix ( $\hat{A}$ and $\hat{B}$ in (19)) in the dynamic inversion baseline controller (termed the inversion failure); an artificial cross coupling of the aircraft ailerons and elevators; a combination of both an inversion failure and an artificial cross coupling; and finally a loss of 50\% of the left horizontal tail, including a loss of the left elevator and the stabilizer. These failures along with various levels of turbulence, provide the basis for the sensitivity analysis presented in the subsequent section.

\section{Sensitivity Analysis and Results}

This section presents results from a sensitivity analysis of each adaptive control technology. The results presented here are for each controller operating with the designer selected gains that achieved the required timedelay robustness. This analysis was performed by evaluating each controller's performance with the damage and failure conditions discussed in section IV.D. Additional discussion of the results for each adaptive control technology is also provided, including comments regarding the ease and/or complexity associated with integration of each technology.

\section{A. Generalized Results}

For the preliminary tuning, the design requirement was that each controller be robust to a .05 second time-delay. Each adaptive controller was then hand tuned such that it met this requirement while still minimally matching the performance of the non-adaptive baseline dynamic inversion controller with an identical time-delay. The results of this preliminary tuning are presented below in Fig. 10. 


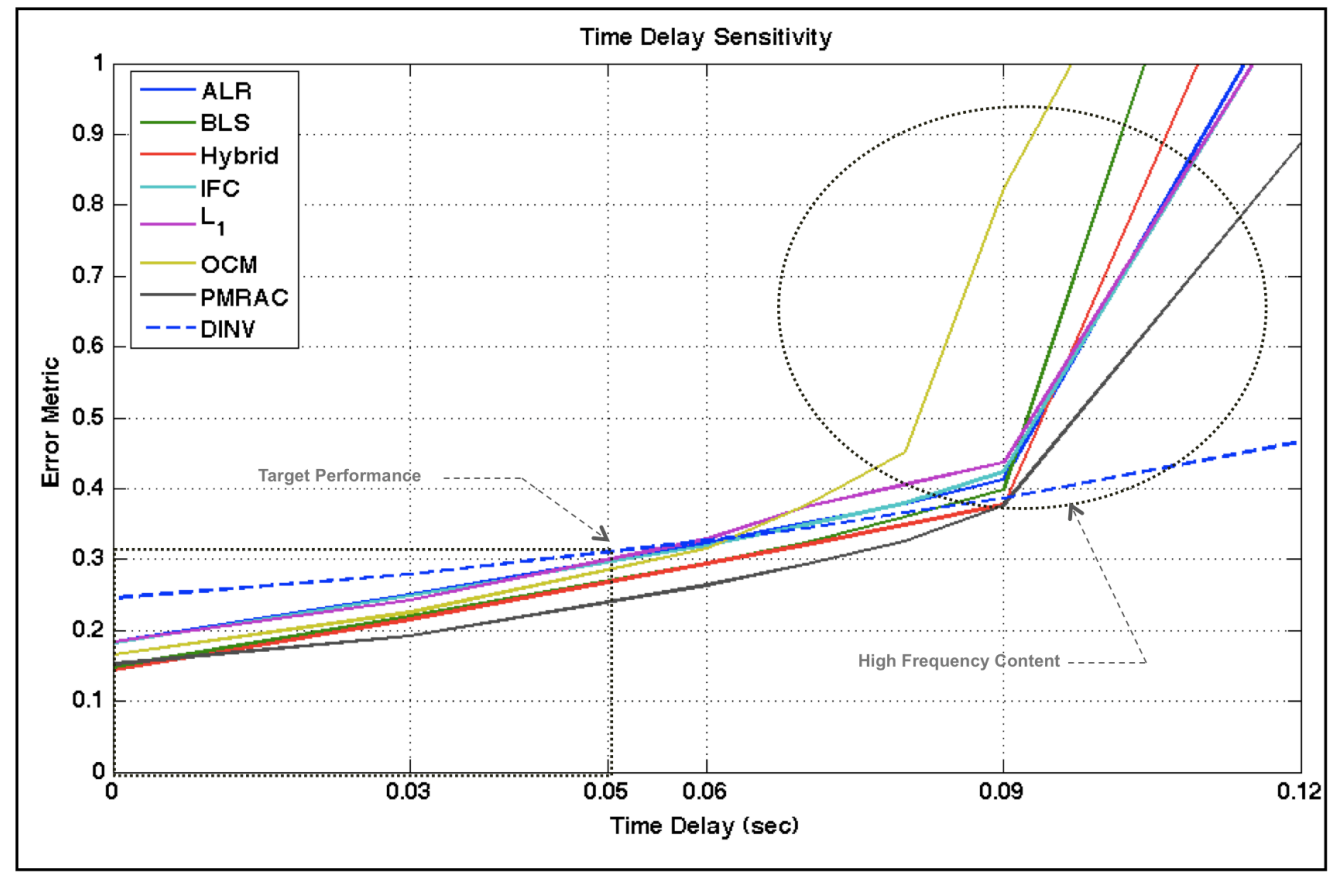

Fig. $10 M_{5}$ error metric for control systems performing standardized lateral, longitudinal, directional doublet (see Fig. 6) with various time-delays (see Fig. 5) at an IAS of $250 \mathrm{Kts}$. and 10,000 ft.

Having established a tuning for each adaptive controller, as shown in Fig. 10, these tunings were fixed for all additional analysis. The first primary analysis was to evaluate the performance of each adaptive controller over a large range of flight conditions. This included adjusting speed and altitude while considering flap deployment. The results of this analysis are presented below in Fig. 11.

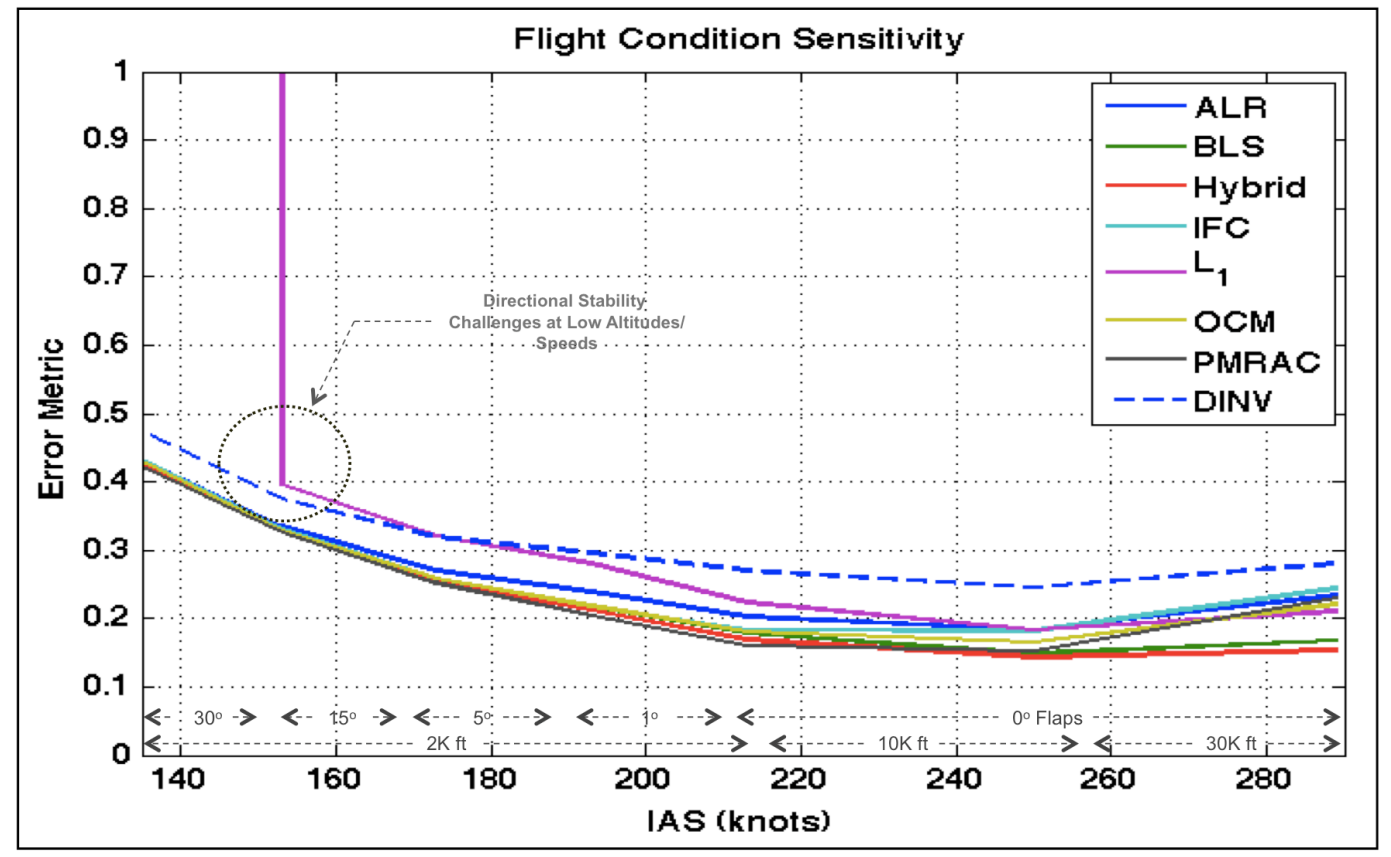

Fig. $11 M_{5}$ error metric for control systems performing standardized lateral, longitudinal, directional doublet (see Fig. 6) at various flight conditions (see Fig. 5). 
To test sensitivity to model uncertainty, we can leverage the dynamic inversion controller and insert large errors in the $\hat{A}, \hat{G}$, and $\hat{B}$ matrices of (19). In particular, the $\hat{A}$ and $\hat{G}$ matrices are removed from the inversion control law and the $\hat{B}$ matrices are scaled by powers of 2 . The resulting analysis is presented below in Fig. 12.

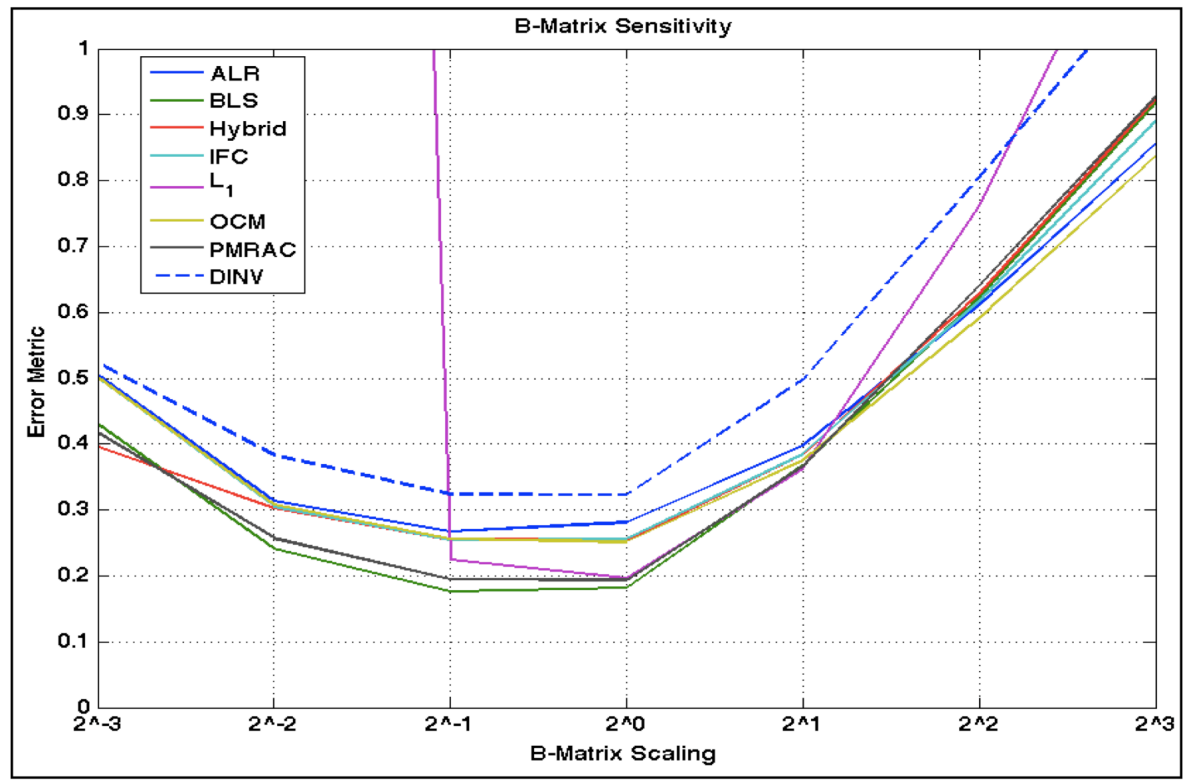

Fig. $12 M_{5}$ error metric for control systems performing standardized lateral, longitudinal, directional doublet (see Fig. 6) with dynamic inversion uncertainty at an IAS of $250 \mathrm{Kts}$. and 10,000 ft.

Cross-coupling sensitivity was explored by inducing a pitch-roll cross-coupling. This was achieved by artificially adding a portion (as dictated by a multiplying factor) of the lateral control signal to the longitudinal control signal. The $M_{5}$ metric is applied separately to the roll and pitch axis for the results presented below.

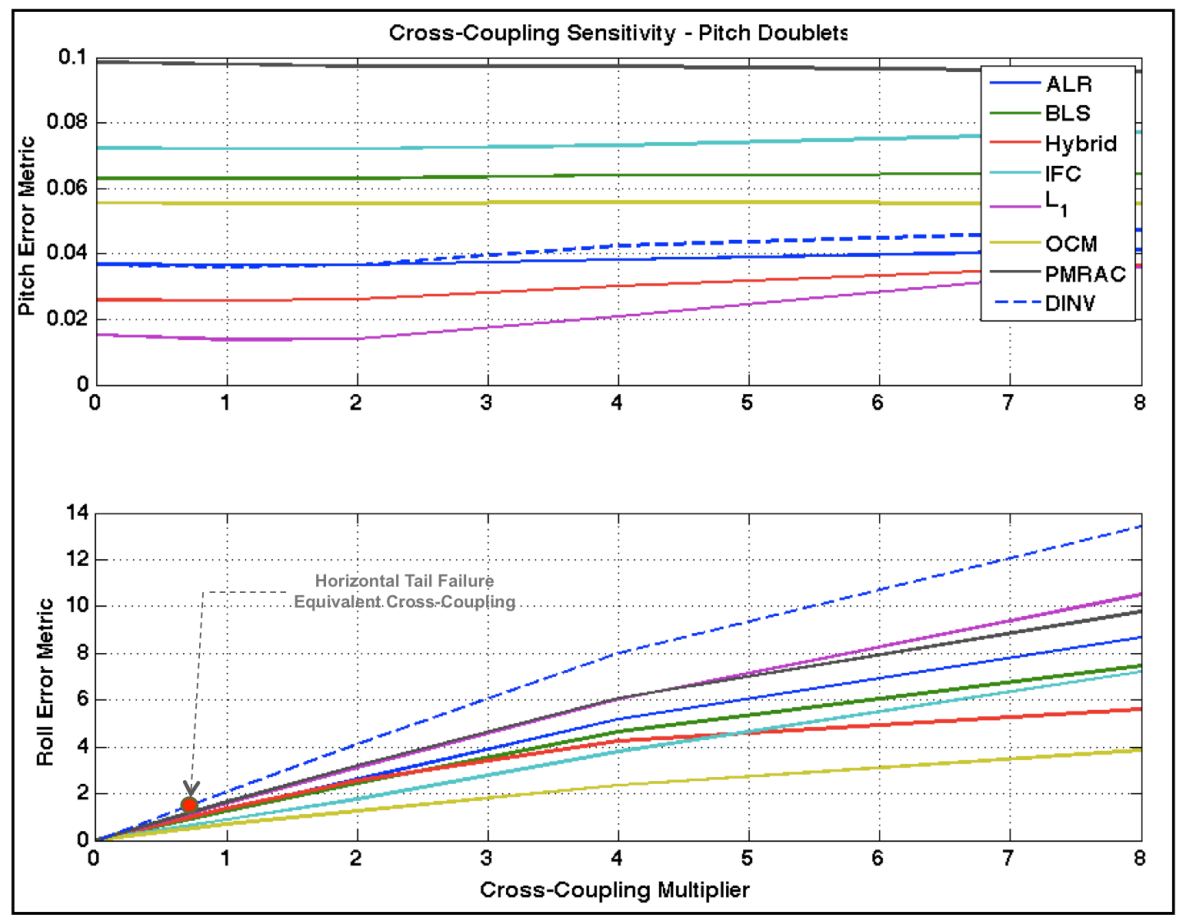

Fig. $13 M_{5}$ error metric for control systems performing standardized longitudinal doublet maneuver with artificial cross-coupling at an IAS of $250 \mathrm{Kts}$. and $10,000 \mathrm{ft}$. 


\section{B. Technology Specific Observations}

In general, the results indicate that the variation amongst the adaptive control technologies is largest with regard to cross-coupling. With regard to the other simulation results, most of the adaptive controllers were able to out perform the baseline dynamic inversion controller. Moreover, the adaptive controllers as a whole demonstrated similar trends and, in many cases, comparable performance. The remainder of this section provides some observations on integrating the respective technologies.

\section{Adaptive Loop Recovery MRAC (ALR)}

The ALR controller is based upon the traditional MRAC approach but adds an additional relative gain term and requires the calculation of partial derivatives. This further complicates the tuning and integration process beyond traditional MRAC. The performance of the ALR controller is dictated in large part by the selection of the relative gain term $v$ in (24). The optimal choice for this term is not necessarily obvious.

\section{Bounded Linear Stability MRAC (BLS)}

The BLS control architecture requires the use of sliding time windows to collect and store state information. This information is then used to calculate an adaptive gain that will preserve the system's stability margins. The integration of this approach requires the storage of additional information, the implementation of additional calculations, and the tuning of the sliding time window duration.

\section{Hybrid Adaptive MRAC (Hybrid)}

The hybrid adaptive controller requires the integration of an indirect adaptive technique. The associated challenges of this are documented in [24]. In general, it is very easy to implement this system in such a way that the indirect adaptive technique dominates and produces artificially impressive results. When incorporating a time-delay approach can breakdown unless the RLS algorithm is implemented robustly. Regardless, there are still concerns regarding the implementation of the RLS algorithm when persistence of excitation is lost.

\section{4. $L_{1}$ Adaptive Control $\left(L_{1}\right)$}

The $L_{l}$ controller requires that additional states be propagated and stored as the result of the inclusion of a predictor system. Additionally, the theory of $L_{l}$ requires the use of large adaptive gains to ensure boundedness, this can pose interesting numerical challenges as instability can result from the use of simple numerical integration techniques. Additionally, the $L_{l}$ controller is difficult to tune and evaluate as the system does not explicitly track the reference model, but instead the predictor system. The selection and design of the $C(s)$ filter in (25) impacts both the overall stability of the system and the amount of uncertainty that can be allowed, but also the way in which the predictor system is shaped by the uncertainty. This complicated relationship adds additional design challenges.

\section{Optimal Control Modified MRAC (OCM)}

The OCM controller adds an additional relative gain, but the value of the gain is known a-priori to reside in the range of [0 1]. As such, only a small amount of additional complexity is added to the traditional MRAC control laws. Of the results presented, the OCM performance can be drastically impacted by the choice of this relative gain, just as with the ALR controller.

\section{Predictor-Based MRAC (PMRAC)}

The PMRAC controller, similar to the $L_{l}$ controller, adds additional prediction states and parameters for tuning. The additional tuning parameters shape the prediction system through the term $A_{p}$ in (28).

\section{7. e-Mod MRAC (IFC)}

The $e$-Mod adaptive controller only adds the tuning of an additional relative gain. This gain is nominally tuned to be less than 1 .

\section{Future Piloted Studies and Evaluations}

The work presented here provides the basis for a full-motion simulation study to be conducted using NASA test pilots. A series of 8 pilots will be used to evaluate each of the previously discussed technologies using the wellknown Cooper-Harper scale; this study is to be conducted using an immersive, full-motion simulator located at the 
NASA Ames Research Center. The results of this study will ultimately provide further insight into the strengths and weaknesses of each technology as it pertains to an actual piloted interaction. The necessity of this study is evinced by the presence of handling qualities requirements in FAA certification requirements. Specifically, the FAA has three ratings: Satisfactory, Adequate, and Controllable. A rating requirement is then prescribed for various combinations of the aircraft flight envelope, atmospheric conditions, and system failures based on the probability of occurrence; extremely remote combinations of occurrences are not considered. In the realm of military procurement vehicles, MIL-HDBK-1797A outlines handling qualities specifications for military aircraft. Thus, irrespective of the application, pilot based evaluations of the aircraft are an integral part of maturing any control technology to a realworld platform.

\section{Conclusions}

This paper has presented a study to evaluate a series of adaptive control technologies. Each technology was preliminarily tuned using a decided upon time-delay requirement. The resulting tuned controllers were then evaluated at a series of flight conditions and under a series of varying failure conditions. The results of this study are presented to the community for consideration along with a series of observations based on the relative challenges of implementing/integrating each adaptive controller. The goal of this study was to present the strengths and weakness associated with each controller by applying them to a consistent baseline control framework. Interestingly, many of the controllers gave comparable performance across a wide variety of failure and flight conditions; the greatest discrepancy in performance was found in the cross-coupling sensitivity analysis. A key point is that adaptive controller performance should not be compared using identical gains, i.e. evaluating an $L_{1}$ adaptive controller against an OCM controller at an identical flight condition with an identical adaptive gain is not insightful. Instead, gains for each controller under consideration should be tuned to meet a specific design requirement and then performance should be compared across a variety of conditions. Ultimately, this will serve as a better indicator of the value of a technology in terms of a complexity versus improved performance.

\section{Acknowledgments}

Special thanks must be given to Gordon Hardy for providing his export opinion and evaluations. Thanks in advance must be given to all the pilots who will participate in the study. Finally, NASA Adaptive Control and Evolvable Systems (ACES) team members Greg Larchev, Shivanjli Sharma, and Susan Frost must be thanked and acknowledged for their contributions to this work.

\section{References}

[1] Cooper, G.E., and Harper, R.P., "The Use of Pilot Rating in the Evaluation of Aircraft Handling Qualities," NASA TN D-5153, April 1969.

[2] Bailey, R. E., Jackson, E. B., Bilimoria, K. D., Mueller, E. R., Frost, C. R., and Alderete, T. S., "Cooper-Harper Experience Report for Spacecraft Handling Qualities Applications," NASA/TM-2009-215767.

[3] Blake, M. W., "The NASA Advanced Concepts Flight Simulator - A Unique Transport Aircraft Research Environment," AIAA Flight Simulation Technologies Conference, San Diego, CA, July 1996.

[4] Jordan, T.L., Langford, W.M., et al., "Development of a Dynamically Scaled Generic Transport Model Testbed for Flight Research Experiments," AUVSI Unmannded Systems North America 2004, Arlington, VA, 2004.

[5] Murch, A.M., and Foster, J.V., "Recent NASA Research on Aerodynamic Modeling of Post-Stall and Spin Dynamics of Large Transport Airplanes," $45^{\text {th }}$ AIAA Aerospace Sciences Meeting and Exhibit, Reno, NV, Jan. 8-11, 2007.

[6] Totah, J. J., Kinney, D. J., Kaneshige, J. T., and Agabon, S., “An Integrated Vehicle Modeling Environment," AIAA Atmospheric Flight Mechanics Conference and Exhibit, Portland, OR, Aug. 9-11, 1999.

[7] Kaneshige, J., Bull, J., and Totah, J.J., "Generic Neural Flight Control and Autopilot System," AIAA Guidance, Navigation, and Control Conference, Denver, CO, Aug 14-17, 2000.

[8] Kaneshige, J., and Burken, J., "Enhancements to a Neural Adaptive Flight Control System for a Modified F-15 Aircraft," AIAA Guidance, Navigation and Control Conference, Honolulu, HI, Aug. 18-21, 2008.

[9] Rysdyk, R. T., and Calise, A. J., "Fault Tolerant Flight control via Adaptive Neural Augmentation," AIAA Guidance, Navigation, and Control Conference, Aug. 1998.

[10] Nguyen, N., Krishnakumar, K., Kaneshige, J., and Nespeca, P., "Flight Dynamics and Hybrid Adaptive Control of Damaged Aircraft," AIAA Journal of Guidance, Control, and Dynamics, Vol. 31, No. 3, pp. 751-764, 2008. 
[11] Snell, S. A., Enns, D. F., and Garrard, W. L., “ Nonlinear Inversion Flight Control for a Supermaneuverable Aircraft," Journal of Guidance, Control, and Dynamcs, Vol. 15, No. 4, July-August 1992.

[12] Calise, A. J., Yucelen, T., and Muse, J. A., “A Loop Recovery Method for Adaptive Control,” AIAA Guidance, Navigation, and Control Conference, Chicago, Illinois, Aug. 2009.

[13] Nguyen, N.T., and Boskovic, J. D., "Bounded Linear Stability Margin Analysis of Nonlinear Hybrid Adaptive Control Conference, Seattle, WA, 2008.

[14] Bakhtiari-Nejad, M., Nguyen, N.T., and Krishnakumar, K., "Adjustment of Adaptive Gain with Bounded Linear Stability Analysis to Improve Time-Delay Margin for Metrics Driven Adaptive Control," AIAA Infotech@aerospace Conference, Seattle, WA, 2009.

[15] Cao, C., and Hovakimyan, N., "Design and Analysis of a Novel $\mathrm{L}_{1}$ Adaptive Controller, Part I: Control Signal and Asymptotic Stability," Proceedings of the 2006 American Control Conference, Minneapolis, MN, June 1416, 2006.

[16] Cao, C., and Hovakimyan, N., "Design and Analysis of a Novel $\mathrm{L}_{1}$ Adaptive Controller, Part II: Guaranteed Transient Performance," Proceedings of the 2006 Amrican Control Conference, Minneapolis, MN, June 14-16, 2006.

[17] Cao, C., and Hovakimyan, N., "Novel $\mathrm{L}_{1}$ Neural Network Adaptive Control Architecture with Guaranteed Transient Performance," IEEE Transactions on Neural Networks, Vol. 18, No 4., July 2007.

[18] Nguyen, N.T., Krishnakumar, K., and Boskovic, J.D., "An Optimal Control Modification Model-Reference Adaptive Control for Fast Adaptation," AIAA Guidance, Navigation, and Control Conference, Honolulu, HI, August 2008.

[19] Lavretsky, E., Gadient, R., and Gregory, I.M., "Predictor-Based Model Reference Adaptive Control," AIAA Guidance, Navigation, and Control Conference, Chicago, IL, Aug. 10-13, 2009.

[20] Dydek, Z. T., Jain, H., Jang, J., and Annaswamy, A., "Theoretically Verifiable Stability Margins for an Adaptive Controller," AIAA Guidance, Navigation, and Control Conference and Exhibit, Keystone, CO, Aug. 21-24, 2006.

[21] Stepanyan, V., Krishnakumar, K., and Nguyen, N.T., "Stability and Performance Metrics for Adaptive Flight Control," AIAA Guidance, Navigation, and Control Conference, Chicago, IL, Aug. 10-13, 2009.

[22] Campbell, S.F., Kaneshige, J., "A Nonlinear Dynamic Inversion $\mathrm{L}_{1}$ Adaptive Controller for a Generic Transport Model," American Control Conference, Baltimore, MD, June 2010.

[23] Campbell, S.F., Kaneshige, J., "A Nonlinear Dynamic Inversion Predictor-Based Model Reference Adaptive Controller for a Generic Transport Model," American Control Conference, Baltimore, MD, June 2010.

[24] Campbell, S.F., Nguyen, N.T., Kaneshige, J.T., and Krishnakumar, K., "Parameter Estimation for a Hybrid Adaptive Flight Controller,” AIAA Infotech@Aerospace Conference, Seattle, WA, April 2009. 